\title{
Erratum to: Group harmony in the workplace: Conception, measurement, and validation
}

\author{
Chao C. Chen ${ }^{1,2}$ • Ali F. Ünal ${ }^{3}$ Kwok Leung ${ }^{4}$. \\ Katherine R. Xin ${ }^{5}$
}

Published online: 9 March 2016

(C) Springer Science+Business Media New York 2016

\section{Erratum to: Asia Pacific Journal of Management DOI 10.1007/s10490-016-9457-0}

During the printing of the original version of the article some unfortunate errors occurred.

Table 1: Item \#4 "AFF - Have feelings of safety and securityx" contained a typo in securityx, $\mathrm{x}$ is now deleted.

The online version of the original article can be found at http://dx.doi.org/10.1007/s10490-016-9457-0.

Chao C. Chen

chaochen@business.rutgers.edu

Ali F. Ünal

afunal@uludag.edu.tr

Kwok Leung

leungkwok@baf.cuhk.edu.hk

Katherine R. Xin

katherinexin@ceibs.edu

1 Department of Management and Global Business, Rutgers, The State University of New Jersey, New Brunswick, NJ, USA

2 Nanjing University Business School, Nanjing University, Nanjing, China

3 Faculty of Economics and Administrative Sciences, Uludağ University, Bursa, Turkey

4 Department of Management, Chinese University of Hong Kong, No.12, Chak Cheung Street, Shatin, N.T., Hong Kong

5 China Europe International Business School (CEIBS), 699 Hongfeng Road, Pudong, Shanghai 201206, People's Republic of China 
Table 4: 1) in Steps 3 and 4 the beta weights $\left(.06^{* *}\right.$ and $.07 * *$ respectively) were

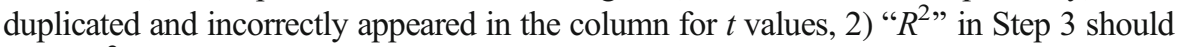
be " $\Delta R^{2}$ ". The original version is now corrected and the correct Table 4 is shown below.

Table 4 Results of hierarchical regression analysis for the interaction of harmony and task conflict $(n=97$ groups)

\begin{tabular}{|c|c|c|c|}
\hline & & Team inno & \\
\hline & & $b$ weight & $t$ \\
\hline Step 1 & Group size & -.02 & -.38 \\
\hline & Age & -.03 & -1.27 \\
\hline & Education & $.75 * * *$ & 3.49 \\
\hline & Organizational tenure & .01 & .34 \\
\hline & Age heterogeneity & -1.92 & -1.25 \\
\hline & Gender heterogeneity & -.54 & -.94 \\
\hline & Tenure heterogeneity & .47 & 1.36 \\
\hline & Education heterogeneity & .42 & .74 \\
\hline & Position heterogeneity & -.41 & -.88 \\
\hline & Function heterogeneity & -.20 & -.56 \\
\hline & Firm size & .07 & .74 \\
\hline & Firm type & .14 & .65 \\
\hline & Firm ownership (d1) & .19 & .71 \\
\hline & Firm ownership (d2) & .03 & .11 \\
\hline & $R^{2}$ & $.22^{\dagger}$ & \\
\hline Step 2 & & & \\
\hline & Harmony & $.34^{\dagger}$ & 1.73 \\
\hline & $\Delta R^{2}$ & $.03^{\dagger}$ & \\
\hline Step 3 & & & \\
\hline & Task conflict & $-.42 *$ & -2.67 \\
\hline & $\Delta R^{2}$ & $.06^{* *}$ & \\
\hline Step 4 & & & \\
\hline & Harmony $\times$ Task conflict & $.87^{* *}$ & 2.94 \\
\hline & $\Delta R^{2}$ & $.07 * *$ & \\
\hline$R^{2}$ & .38 & & \\
\hline Adjusted $R^{2}$ & .25 & & \\
\hline$F(17,79)$ & $2.88^{* *}$ & & \\
\hline
\end{tabular}

